\title{
Development of a Broadband Triboelectric Energy Harvester With SU-8 Micropillars
}

\author{
Lokesh Dhakar, Francis Eng Hock Tay, and Chengkuo Lee, Member, IEEE
}

\begin{abstract}
This paper describes a broadband energy harvester working on the principle of contact electrification or triboelectric charging. Design and fabrication of the device have been discussed. The device uses contact and separation mechanism using a cantilever to generate triboelectric charges. This mechanism introduces nonlinearity in the cantilever, which results in broadband behavior of triboelectric energy harvester. The device uses SU-8 micropillar arrays to enhance the triboelectric charging. A study is conducted to study the effect of the micropillar sizes on the power output of devices. The devices were tested at different acceleration levels. The peak power output achieved is $0.91 \mu \mathrm{W}$ at an acceleration of $1 \mathrm{~g}$. The amplitude limiter based design of the energy harvester enables broadening of operating bandwidth as the acceleration level increases. A maximum operating bandwidth of $22.05 \mathrm{~Hz}$ was observed at $1.4 \mathrm{~g}$ increasing from an operating bandwidth of $9.43 \mathrm{~Hz}$ at $0.4 \mathrm{~g}$.

[2013-0401]
\end{abstract}

Index Terms-Broadband behavior, energy harvesting, amplitude limiter, tribolelectricity.

\section{INTRODUCTION}

$\mathbf{H}$ ARVESTING mechanical energy is one of the most important sources of energy to power wireless sensor nodes [1]-[6] and low power electronic equipments [7], [8] as it is least affected by the whims of environment such as sunlight, temperature conditions or location. Traditionally, mechanical energy harvesters have used different mechanisms namely piezoelectric [9]-[15], electromagnetic [16]-[20] and electrostatic [21]-[24] mechanism for power generation. These mechanisms use special material properties like piezoelectricity or magnetism to harvest mechanical energy. Therefore, use of these mechanisms poses significant limitation

Manuscript received December 29, 2013; revised March 17, 2014; accepted April 2, 2014. Date of publication May 5, 2014; date of current version January 30, 2015. This work was supported in part by the Faculty Research Committee under Grant R-263-000-692-112 through the National University of Singapore, Singapore, and in part by the NRF2011 NRF-CRP001-057 Program Self-Powered Body Sensor for Disease Management and PreventionOrientated Healthcare under Grant R-263-000-A27-281 through the National Research Foundation, Singapore. Subject Editor X. Wang.

L. Dhakar is with the Department of Electrical and Computer Engineering, National University of Singapore, Singapore 117576, and also with the NUS Graduate School of Integrative Sciences and Engineering, National University of Singapore, Singapore 117576 (e-mail: lokesh@nus.edu.sg).

F. E. H. Tay is with the Department of Mechanical Engineering, National University of Singapore, Singapore 117576, and also with the Graduate School for Integrative Sciences and Engineering, National University of Singapore, Singapore 117576 (e-mail: mpetayeh@nus.edu.sg).

C. Lee is with the Department of Electrical and Computer Engineering, National University of Singapore, Singapore 117576 (e-mail: elelc@nus.edu.sg).

Color versions of one or more of the figures in this paper are available online at http://ieeexplore.iee.org.

Digital Object Identifier 10.1109/JMEMS.2014.2317718 on the materials which can be used for harvesting energy. Recently, tribolelectricity has been investigated as a possible mechanism for harvesting mechanical energy [25]-[29]. Triboelectric mechanism uses the process of contact electrification and electrostatic induction to convert mechanical energy into useful electrical energy. Contact electrification [30], [31] is the phenomenon of electrification or charging by contact of two dissimilar surfaces. This phenomenon occurs when two materials are put into contact and separated. The difference in work function of the materials is the reason for different tendencies of different materials to attract or donate electrons [32]. These materials can be arranged in order known as triboelectric series according to their tendency to donate or attract electrons [33]. The fundamental mechanism behind the contact electrification is not fully understood. Although there has been a lack of one overarching model to explain contact electrification and charge transfer, several models and theories have been reported to study contact electrification between various materials [34].

One of the major problems which most of the resonant mechanism based energy harvesting devices suffer from is the operating bandwidth. The resonant mechanism based devices have the capability to operate only in a narrow range of bandwidth near the resonant frequency. Many of the researchers have studied this problem in piezoelectric and electromagnetic mechanism based energy harvesting devices. Sari et. al. [35] used an array of cantilevers with different dimensions to achieve broad range of operating bandwidth. Tvedt et al. [36] used non-linear springs under colored noise vibrations which resulted in broadband behavior. Frequency tuning mechanism was demonstrated by Challa et al. [37] by applying a magnetic force on a cantilever in transverse direction. Triboelectric mechanism based resonant energy harvesters also face the same problem of narrow operating bandwidth. The current design for triboelectric energy harvester (TEH) utilizes the basic contact and separation motion used in triboelectric mechanism to induce non-linearity in spring constant leading to broadening of the operating bandwidth of device [38], [39]. This work is an important step towards design concept of vibration based energy harvesting devices based on the principle of contact electrification. It presents a solution to the problem of narrow operating bandwidth in energy harvesting devices. The device presented in this work uses SU-8 micropillars to enhance the triboelectric generation. A study has also been conducted to examine the effect of micropillar array configuration on the power generation characteristics of the device. 


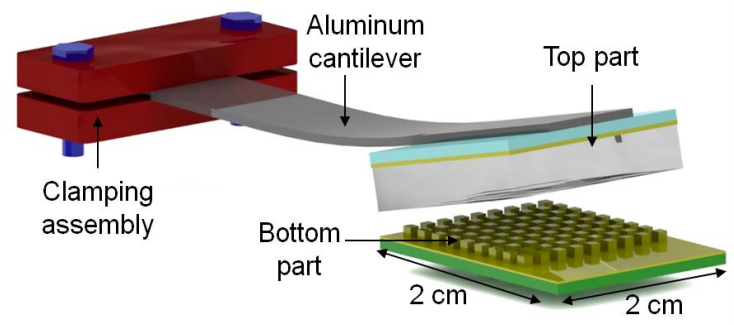

Fig. 1. Schematic of the triboelectric energy harvester.

\section{DESIGN AND FABRiCATION}

\section{A. Design and Device Configuration}

The schematic of the proposed device using triboelectric mechanism is shown in Fig. 1. The as-fabricated TEH comprises of two parts: i) top part and ii) bottom part. The device is based on the periodic contacting and separating motion between top and bottom parts. The top part consists of a gold thin film on a glass substrate with polydimethylsiloxane (PDMS) layer on top of it. The bottom part constitutes of an array of SU-8 micropillars on silicon substrate which has gold film coated on top of it. The top part is attached to an aluminum cantilever which is fixed using clamping assembly at one end (Fig. 1). The bottom part is kept fixed when the device is in operation. The periodic contacting and separating motion between top and bottom parts is realized by the vibrating motion of cantilever when excited by mechanical vibrations. In practical applications, these vibrations can be obtained from human motion [18], household equipments [6], machine vibrations etc. During the contacting-separating motion, the bottom part acts as an amplitude limiter for cantilever motion which induces non-linearity resulting in broadband behavior of the cantilever based resonant TEH [38]-[42].

\section{B. Fabrication Process}

The steps involved in the fabrication of top and bottom parts are shown in Fig. 2. Fabrication of top part starts with preparation of the glass substrate. A $100 \mathrm{~nm}$ thick layer of gold is then coated on the glass substrate using thermal evaporation. This gold layer serves as the top electrode for TEH. On top of gold film, a $500 \mu \mathrm{m}$ thick PDMS layer is spin coated using SYLGARD 184 silicone elastomer kit. The spin coated PDMS layer is then kept in oven at $80^{\circ} \mathrm{C}$ for 2 hours for curing. The PDMS layer acts as an dielectric layer through which the gold electrode gets charged by electrostatic induction mechanism. The top part is then attached to an aluminum cantilever as shown in Fig. 1 using epoxy adhesive.

The bottom part fabrication starts with preparation of silicon substrate. A $50 \mu \mathrm{m}$ thick layer of negative photoresist SU-8 2025 from MicroChem is then spin coated on the silicon substrate. It is then soft baked in steps at $65^{\circ} \mathrm{C}$ and $95{ }^{\circ} \mathrm{C}$ for 3 minutes and 7 minutes, respectively. SU-8 is then exposed for patterning using photolithography. Thereafter post exposure bake is done. The samples are then developed using MicroChem's SU-8 developer which results in SU-8 micropillar arrays as shown in Fig. 2(b) and 2(c).
The micropillar arrays are then hard baked to improve the mechanical properties. The SU-8 micropillars are then coated with a $100 \mathrm{~nm}$ thick of gold which serves as the bottom electrode for TEH.

\section{Theoretical Modeling}

\section{A. Mechanics Modeling}

A cantilever is used for contact-separation motion to generate charge using contact electrification process. This section discusses the mechanics of top part attached to the vibrating cantilever. The cantilever is excited with a sinusoidal mechanical vibration using an electromagnetic shaker. TEH cantilever vibrating motion can be modeled as a forced, damped spring mass system. The equation for forced vibrations of damped spring mass system can be written as:

$$
m \ddot{y}+c \dot{y}+k y=F_{0} \sin (\omega t)
$$

The steady state solution for the system defined by (1) can be given by:

$$
y=\frac{F_{0}}{\sqrt{\left(k-m \omega^{2}\right)^{2}+(c \omega)^{2}}} \sin (\omega t-\phi)
$$

where $\phi=\tan ^{-1}\left(\frac{c \omega}{k-m \omega^{2}}\right), m$ is mass, $c$ is damping coefficient, $k$ is the spring constant, $F_{0}$ is the amplitude of the sinusoidal excitation force, $\omega$ is the excitation frequency. Differentiating (2) with respect to time, the velocity function of the mass can be obtained as in (3).

$$
\dot{y}=\frac{F_{0} \omega}{\sqrt{\left(k-m \omega^{2}\right)^{2}+(c \omega)^{2}}} \cos (\omega t-\phi)
$$

If the excitation frequency of the shaker matches the resonant frequency $\left(\omega_{\mathrm{r}}\right)$ of the shaker, from (3) the equation of motion can be written as:

$$
\dot{y}=\frac{F_{0}}{c} \cos \left(\omega_{r} t-\phi\right)
$$

Fig. 3 shows the cantilever beam vibrating with the frequency $\omega$ impacting the bottom part. The average impact force (F) is generated due to the change in momentum as expressed in (5).

$$
F=\frac{\text { change in momentum }}{\Delta T}=\frac{\left(m \cdot v_{n}-0\right)}{\Delta T}=\frac{m \dot{y}}{\Delta \mathrm{T}}
$$

where $v_{n}$ is the normal component of velocity for the top part and $\Delta T$ is the duration of impact. In (5), for the force calculation it is assumed that the top part comes to rest after impacting the bottom part. After plugging the value of $\dot{y},(5)$ can be written as:

$$
F=\frac{m F_{0}}{c \Delta T} \cos \left(\omega_{r} t-\phi\right)
$$

This is a simplistic model for the impact force between top and bottom parts. It models the vibrating cantilever as a damped spring mass system and assumes that the top part comes to rest after impacting the bottom part. The local deformation for PDMS layer and individual SU-8 micropillar can be approximately modeled using the Hertz Theory [43]. Keeping all the geometrical parameters and material properties same, 
Top part

2(a) 1. Glass substrate 2. Au coating 3. PDMS preparation

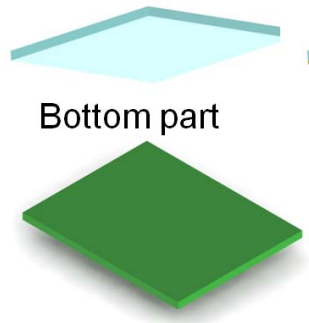

1. Silicon substrate
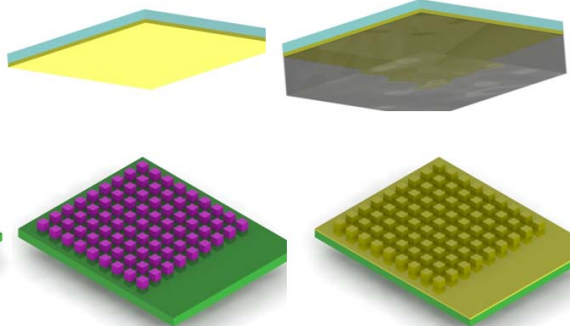

2. SU-8 pillars

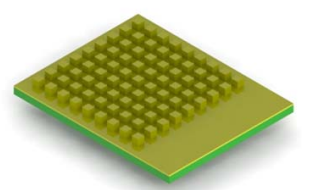

3. Au coating
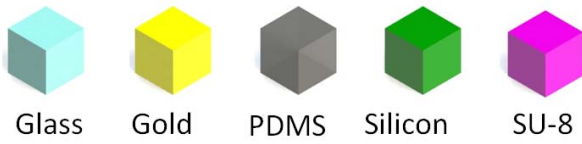

SU-8

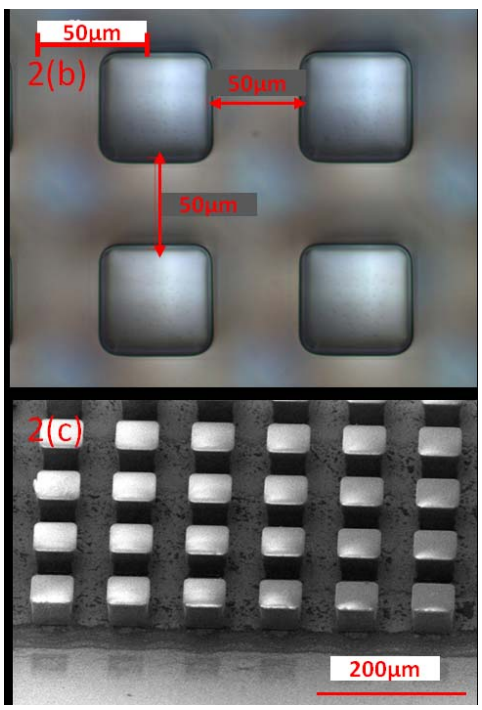

Fig. 2. (a) Steps involved in the fabrication of top and bottom part. (b) Optical image of SU-8 micropillars for cross section of $50 \mu \mathrm{m} \times 50 \mu \mathrm{m}$. (c) SEM micrograph of array of SU-8 micropillars of dimension $50 \mu \mathrm{m} \times 50 \mu \mathrm{m} \times 50 \mu \mathrm{m}$.

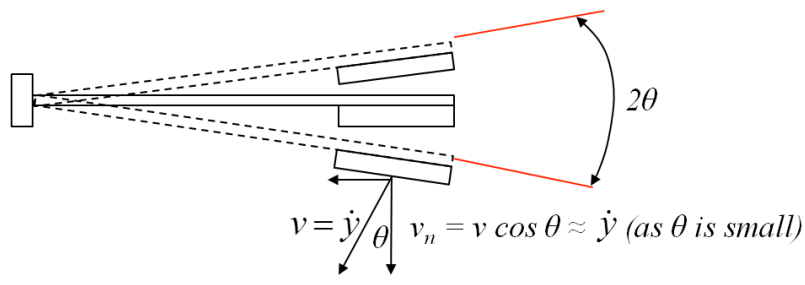

Fig. 3. Calculation of impact force between the top and bottom part.

the relation between duration of contact and impact velocity is given by [43], [44]:

$$
\Delta T \propto \dot{y}^{-1 / 5}
$$

where $\dot{y}$ is the impact velocity. From (6) and (7), following relation can be observed:

$$
F \propto F_{0}^{6 / 5}
$$

It is clear from (8) that as the magnitude of the excitation force or acceleration is increased, the impact force between top and bottom parts increases. Although the model used for impact force is simplified, it serves the purpose of understanding the qualitative relation between the magnitude of excitation force and impact force between top and bottom parts.

\section{B. Deformation in PDMS and SU-8 Pillars}

The micropillar array can be assumed to be an array of springs fixed at one end but free at the other. Hooke's law is used to calculate the spring constant of the SU-8 micropillars, as Euler theory cannot be applied due to the slender shape assumption [45]. The spring constant by Hooke's law is given by following equations:

$$
\begin{aligned}
\sigma & =E \varepsilon \\
\frac{F}{A} & =E \frac{\Delta h}{h} \\
k & =\frac{F}{\Delta h}=\frac{E A}{h}
\end{aligned}
$$

TABLE I

PARAMETERS For SPRING Constant CALCUlation

\begin{tabular}{ccc}
\hline \hline Structure & $\begin{array}{c}\text { Young's } \\
\text { modulus }\end{array}$ & Dimension \\
\hline SU-8 pillar & $\sim 2 \mathrm{GPa}$ & $50 \mu \mathrm{m} \times 50 \mu \mathrm{m} \mathrm{x}$ \\
& & $50 \mu \mathrm{m}$ \\
PDMS & $\sim 500 \mathrm{kPa}$ & $2 \mathrm{~cm} \mathrm{\times} 2 \mathrm{~cm} \mathrm{\times 500}$ \\
layer & & $\mu \mathrm{m}$ \\
\hline \hline
\end{tabular}

where $\sigma$ is stress, $E$ is Young's modulus, $F$ is the force applied in normal direction, $A$ is the cross section area, $h$ is the height of micropillar, $\Delta h$ is the change in height and $k$ is the spring constant.

The spring constant for the micropillars can be calculated using (11). For sample calculation, a micropillar dimension of $50 \mu \mathrm{m} \times 50 \mu \mathrm{m} \times 50 \mu \mathrm{m}$ is used as shown in Table I. The spring constant for individual micropillar is calculated to be $100000 \mathrm{~N} / \mathrm{m}$. All the SU-8 micropillars are connected in parallel during deformation in contact mode. Therefore the effective spring constant for SU-8 can be written as:

$$
k_{S U-8}=n \times k_{S U-8 \text { pillar }}
$$

where $n$ is the total number of pillars in the array.

Using (12) the value of $k_{S U-8}$ is calculated to be $4 \times 10^{9} \mathrm{~N} / \mathrm{m}$ as $n=40000$ for the micropillar array of $50 \mu \mathrm{m} \times 50 \mu \mathrm{m}$ pillars. The spring constant for the PDMS layer can also be obtained using (11) to be $4 \times 10^{5} \mathrm{~N} / \mathrm{m}$.

When SU-8 micropillar array and the PDMS layer are in contact, the deformation in both PDMS and SU-8 can be calculated using simple spring in series model as shown in Fig 4(b). The ratio of deformation in the PDMS layer and SU-8 micropillars can be used to understand the effect of layer thickness and micropillar heights on deformation. For two springs in series as shown in Fig. 4(b), the ratio of deformation 


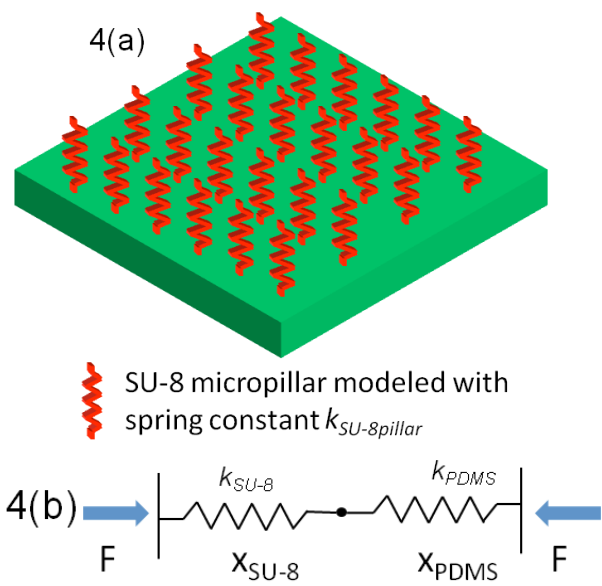

Fig. 4. (a) SU-8 micropillars modeled as linear springs (b). In contact state, deformation PDMS layer and SU-8 micropillars is calculated by spring in series model.

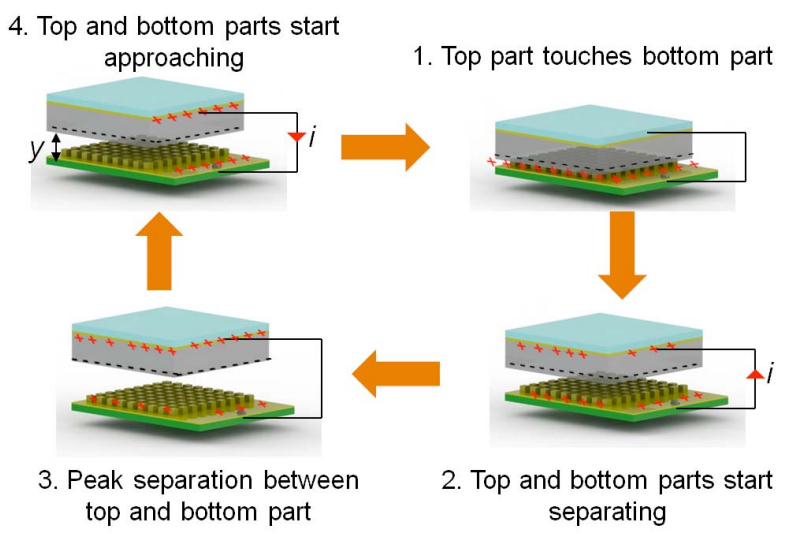

Fig. 5. Working mechanism of TEH at different stages of cantilever vibration.

in two springs can be calculated as:

$$
\frac{x_{P D M S}}{x_{S U-8}}=\frac{k_{S U-8}}{k_{P D M S}}=\frac{4 \times 10^{9}}{4 \times 10^{5}}=10000
$$

As can be seen from (13), during the contact state, most of the deformation occurs in PDMS layer. This is due to high stiffness of SU-8 pillars as compared to the PDMS layer. This indicates that the contact area between SU-8 micropillars and PDMS layers is due to two factors: (i) primary contact area because of micropillar cross section and (ii) contact area resulting due to elastic deformation of PDMS layer. Deformation of SU-8 micropillars does not contribute significantly to the contact area between two materials.

\section{Working Mechanism}

The working mechanism of TEH is described in Fig. 5. Initially, before providing the mechanical excitation, the top and bottom part are in separated position without any prior charges. As the device is mechanically excited with vibrational frequency in operating range, the cantilever starts to vibrate. Thereafter, the distance $y$ as shown in Fig. 5 decreases and top (PDMS) and bottom (gold) parts come in contact with each other. As per triboelectric series, gold has higher tendency to donate electrons as compared to PDMS. Therefore in state 1, when the gold coated on the SU-8 micropillars and PDMS layer are in contact, gold layer gets positively charged whereas the PDMS layer gets negatively charged. Now as the two parts start separating from each other, bottom gold electrode is at higher potential than the top gold electrode. Therefore, electrons start flowing from top gold electrode to bottom gold electrode resulting in current $i$ as shown in state 2 in Fig. 5. This current keeps flowing till the cantilever reaches the other end of its vibration cycle where the separation between top and bottom parts is maximum (state 3). At this point, an electrostatic equilibrium is reached. As the cantilever is vibrating under mechanical excitation, the top part starts approaching (state 4) the bottom part and electrostatic equilibrium between the different layers is disturbed. Now, the current starts flowing from top electrode to bottom electrode which is in the opposite direction of current in the separation motion of two parts. Thereafter, electrostatic equilibrium is reached when the gold layer and PDMS layer are again in contact with each other (state 1). The charges flowing in the external circuit connected to the circuit can be harvested to power wireless sensor nodes or low power electronic devices.

\section{Simulation of Open Circuit Voltage at Different Positions}

A better understanding of the working mechanism of TEH can be gained using the simulation of potential distribution across the electrodes. The simulations were carried out using COMSOL. In the simulations conducted, a charge density of $-10 \mathrm{nC} / \mathrm{m}^{2}$ was assumed on the PDMS layer surface. The results are shown in Fig. 6 for different values of $y$ which is the distance between the top and bottom part. The bottom electrode is taken as reference in the simulation and was grounded for the purpose. As can be seen from the simulation results, the potential difference between the top and bottom electrodes keeps increasing as the two electrodes are separated further away from each other. The potential difference for a distance of $1 \mathrm{~mm}$ is $0.8 \mathrm{~V}$ while it increases to more than $5.8 \mathrm{~V}$ as the distance is increased to $10 \mathrm{~mm}$. So as the top and bottom parts are further separated, mechanical work is done against the electric field due to which the potential difference is increased between the top and bottom electrodes. This results in the flow of electrons from the top electrode to the bottom electrode as the top and bottom parts are separated.

\section{EXPERIMENTS AND DISCUSSION}

\section{A. Experimental Setup}

To study TEH devices, an electromagnetic shaker is used to provide mechanical excitation. The setup for testing the devices is shown in Fig. 7. A software is used to generate sinusoidal signal which is then sent to an amplifier to amplify the signal. The amplified signal is then sent to the electromagnetic shaker which simulates the low frequency vibrations. To measure the acceleration provided by the electromagnetic shaker, an accelerometer is assembled on shaker along with TEH. The accelerometer signal is then again sent to the software which results in a closed loop. This feedback loop is used to maintain the acceleration level provided by the shaker. 


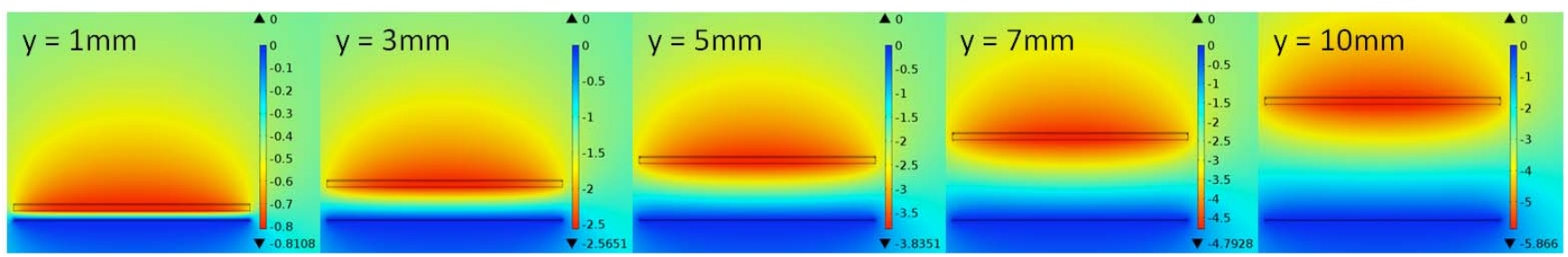

Fig. 6. Potential distribution at different distance between top and bottom parts along the normal direction (' $y$ ' as shown in Fig. 5).

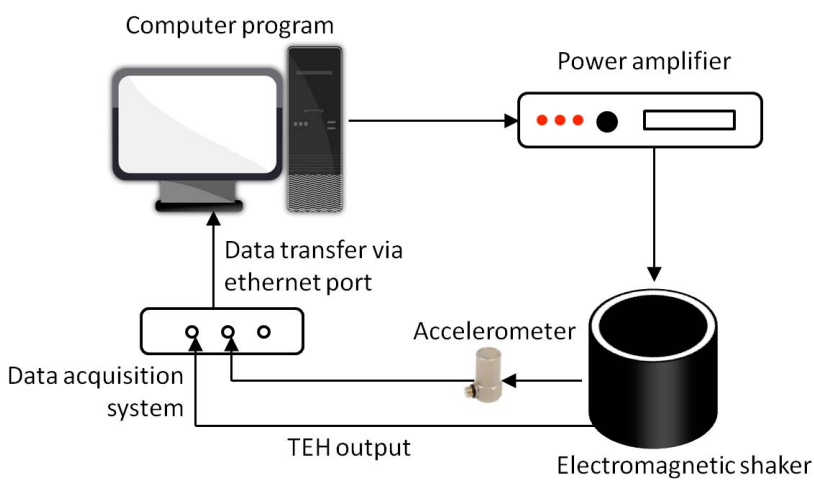

Fig. 7. Experimental setup for testing vibration based TEH.

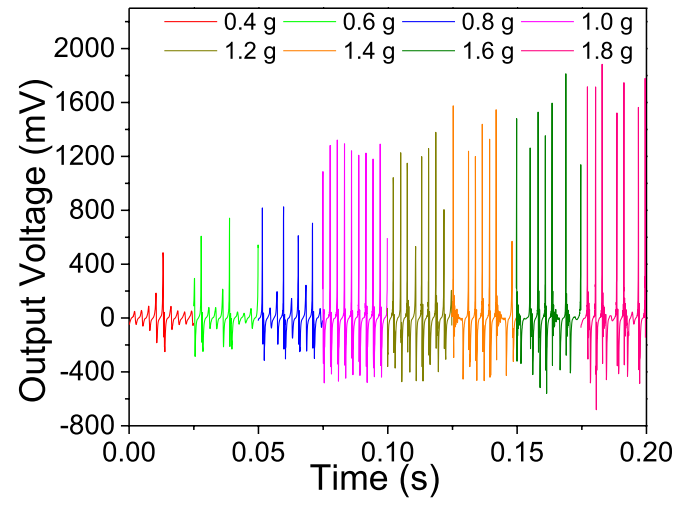

Fig. 8. Output voltage of TEH at different acceleration levels from shaker.

\section{B. Effect of Increasing Acceleration}

The effect of excitation acceleration is studied on the output open circuit voltage generated by TEH by varying the acceleration provided by shaker from $0.4 \mathrm{~g}$ to $1.8 \mathrm{~g}$ in steps of $0.2 \mathrm{~g}$. Although the triboelectric mechanism is not fully understood, one of the parameters which seem to affect the power output was the impact force provided by the top part attached to the vibrating cantilever. As the amplitude of excitation acceleration or force increases, the impact force also keeps increasing as discussed in earlier section and explained by (8). Higher impact force results in increased elastic deformation in the PDMS layer which leads to increased contact area between the two triboelectric layers. The increased contact area is possibly the reason for increased triboelectric generation and performance of the device. Fig. 8 shows the time domain signal for the output voltage at different accelerations. The peak output voltage increases from $450 \mathrm{mV}$ to $1900 \mathrm{mV}$ as the acceleration is increased from $0.4 \mathrm{~g}$ to $1.8 \mathrm{~g}$.
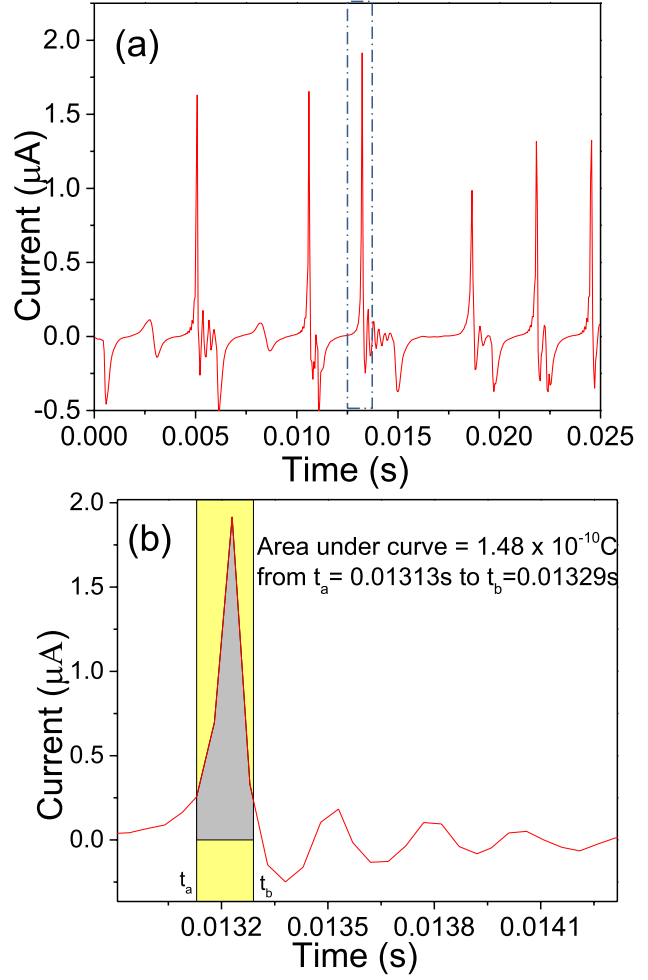

Fig. 9. (a) Current for TEH with a load resistance of $500 \mathrm{k} \Omega$. (b) Zoomed in peak from current signal for the calculation of charge.

\section{Calculation of Charge Density}

During the contact electrification process, triboelectric charges are generated due to which surfaces gets charged resulting in electrostatically induced charges on electrodes. The charge densities on top electrode can be calculated by the calculation of charge by calculating the area under the curve for current versus time graph as shown in Fig.

$$
q=\int_{t_{a}}^{t_{b}} i(t) d t
$$

Fig. 9(a) shows the current for TEH in operation. For the calculation of charge flowing through the external circuit, a peak is selected as highlighted in Fig. 9(a). The magnified graph for the peak is shown in Fig. 9(b). For calculating the charge flown through the external circuit as given by (14), two time points are selected $t_{a}$ and $t_{b}$. The area under the curve for current versus time graph between time $t_{a}$ and $t_{b}$ is calculated to obtain the charge flown. The charge flown through the external circuit between time $t_{\mathrm{a}}$ and $t_{\mathrm{b}}$ is calculated to be $1.48 \times 10^{-10} \mathrm{C}$. The charge density on the top electrode 


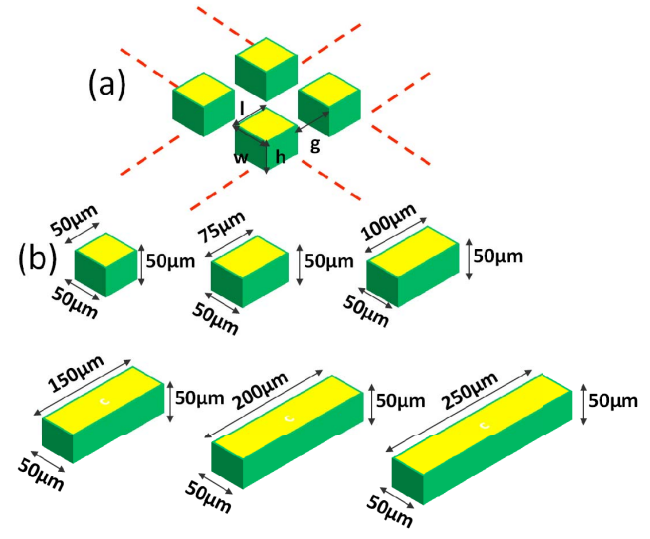

Fig. 10. (a) Micropillar array dimensional parameters. (b) Different pillar dimensions used for preparing SU-8 micropillar arrays.

TABLE II

FILL FACTORS FOR MICROPILLAR ARRAYS

\begin{tabular}{ccc}
\hline \hline Sample number & Pillar size & Fill factor \\
\hline S1 & 50 um x 50 um & 1 \\
S2 & 75 um x 50 um & 1.5 \\
S3 & $100 \mathrm{um} \times 50 \mathrm{um}$ & 2 \\
S4 & $150 \mathrm{um} \times 50 \mathrm{um}$ & 3 \\
S5 & $200 \mathrm{um} \times 50 \mathrm{um}$ & 4 \\
S6 & $250 \mathrm{um} \times 50 \mathrm{um}$ & 5 \\
\hline \hline
\end{tabular}

due to charge flown through external circuit can be calculated as:

$$
\sigma=\frac{q}{\text { Total electrode area }}
$$

The approximate charge density from (15) is calculated as $37 \mathrm{pC} / \mathrm{cm}^{2}$.

\section{Design of Experiment}

To study the effect of micropillar size, six sample variations for the SU-8 micropillar array were prepared. The overall sample size for all six micropillar arrays was kept same to be $2 \mathrm{~cm} \times 2 \mathrm{~cm}$. The micropillar array configuration is shown in Fig. 10(a). In the micropillar arrays, the gap between the micropillars is denoted as $g$. The cross section of an individual micropillar is characterized by the length $l$ and width $w$. The height of the micropillars $h$ and the gap $g$ between individual SU-8 micropillars is kept constant for all the six micropillar arrays fabricated. Different micropillar sizes fabricated for the micropillar arrays are shown in Fig. 10(b). An important parameter to characterize the micropillar arrays is fill factor, which is defined here as:

$$
\text { Fill factor }=l / g
$$

The fill factor defined above characterizes the ratio of length of micropillar and gap between micropillars along the same direction keeping other parameters intact. The fill factor calculated for the six pillar arrays are summarized in Table II. The frequency responses in terms of RMS output voltage for frequency sweep from $10 \mathrm{~Hz}$ to $60 \mathrm{~Hz}$ for acceleration $0.4 \mathrm{~g}$ to $1.6 \mathrm{~g}$ are shown in Fig. 11.

\section{E. Voltage and Power Characteristics}

To calculate the power generated by $\mathrm{TEH}$, a load resistor is connected between the top and bottom electrode. The voltage is then measured across the load resistor to obtain the power generated by the device. As the load resistance is increased, the power output increases and peaks at a point and starts dropping thereafter. Voltage output and power characteristics for sample S6 are shown in Fig. 12. The results have been presented for only sample S6 as the power characteristics for all the samples will follow a similar pattern. The maximum power output is achieved at a load resistance of approximately $400 \mathrm{k} \Omega$. The maximum peak power generated for sample S6 at an acceleration of $1 \mathrm{~g}$ was measured to be $0.91 \mu \mathrm{W}$ at a vibrational excitation frequency of $24.5 \mathrm{~Hz}$. The maximum power density for TEH was calculated to be $0.23 \mu \mathrm{W} / \mathrm{cm}^{2}$.

\section{F. Broadband Behavior of TEH}

The impact between the top and bottom part introduces non-linearity in the cantilever beam spring constant. This non-linearity in the cantilever is expected to increase the operating bandwidth of resonant TEH. To study the broadband behavior of TEH, micropillar arrays S1 to S6 were tested for frequency range $10 \mathrm{~Hz}$ to $60 \mathrm{~Hz}$ for acceleration levels $0.4 \mathrm{~g}$ to $1.6 \mathrm{~g}$ as shown in Fig. 11. As the acceleration level was increased, the operating bandwidth also increased continuously. This behavior was consistently observed across all the micropillar array samples for TEH. The percentage changes in operating bandwidth as the excitation acceleration increased from $0.4 \mathrm{~g}$ to $1.6 \mathrm{~g}$ are summarized in Table III.

\section{G. Effect of Fill Factor on Power Generation}

Fan et al. [28] conducted a study which studied the triboelectric performance of patterned and unpatterned surfaces. The study suggested that patterned films surpassed performance of films without any patterns due to enhanced triboelectric charging. In this paper, we have used the rectangular micropillar shaped structures to enhance the triboelectric charging and studied the effect of different micropillar dimensions on the power generated. Experiments were conducted on micropillar arrays S1 to S6. Peak power generated for different arrays is summarized in Fig. 13. As the fill factor is increased for the micropillar array, the peak power was observed to be increasing but had a diminishing effect as shown in Fig. 13. The increase in power generated is attributed to the increase in contact surface area between the PDMS layer and gold coated SU-8 micropillars. As the contact surface area increases, the contact electrification process is enhanced resulting in higher amount of triboelectric charges. At the same time, the air voids formed between the micropillars also play an important role in generation of triboelectric charges as the separation of the charges between two surfaces becomes easier due to air voids [28]. Therefore the size of air voids between the micropillars affect the performance of TEH. This is also the reason that effect of increasing the micropillar 

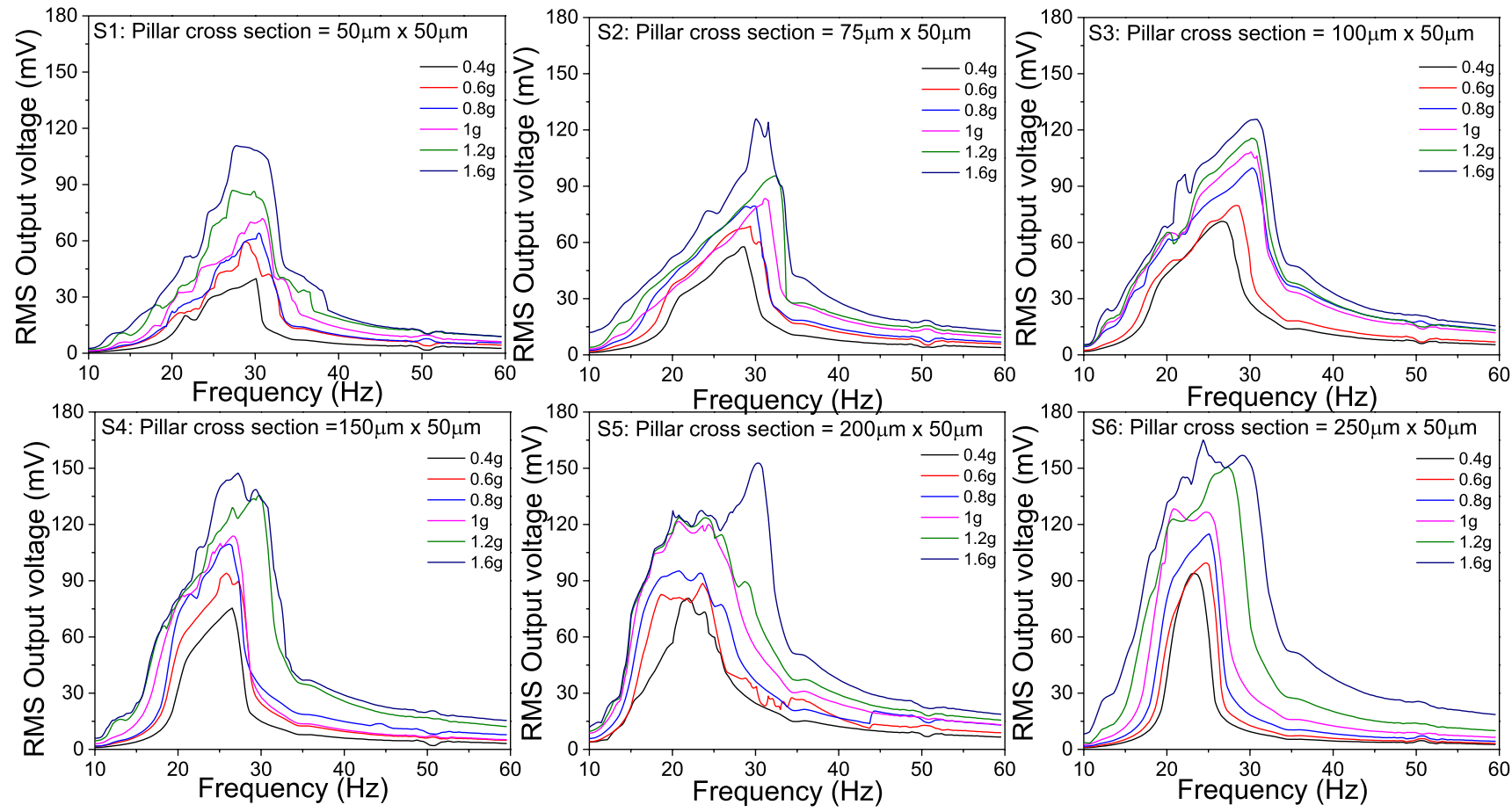

Fig. 11. Frequency response of TEH at for frequency sweep from $10 \mathrm{~Hz}$ to $60 \mathrm{~Hz}$.

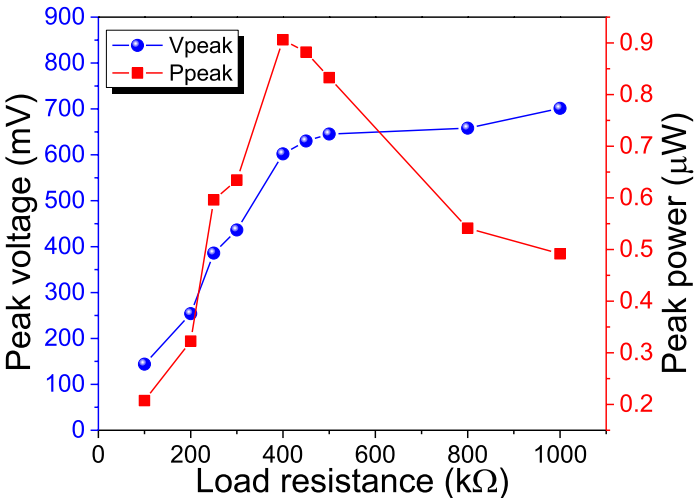

Fig. 12. Peak voltage and peak power characteristics generated using sample S6 at various load resistances at an acceleration of $1 \mathrm{~g}$ and frequency $24.5 \mathrm{~Hz}$

TABLE III

OPERATING BANDWIDTH

\begin{tabular}{ccccc}
\hline \hline Type & $\begin{array}{c}\text { RMS } \\
\text { voltage } \\
\text { level for } \\
\text { bandwidth }\end{array}$ & $\begin{array}{c}\text { Bandwidth } \\
\text { at } 0.4 \mathrm{~g}\end{array}$ & $\begin{array}{c}\text { Bandwidth } \\
\text { at } 1.6 \mathrm{~g}\end{array}$ & $\begin{array}{c}\text { change in } \\
\text { bandwidth }\end{array}$ \\
\hline S1 & $30 \mathrm{mV}$ & 5.36 & 20.5 & $282 \%$ \\
S2 & $35 \mathrm{mV}$ & 7.64 & 21.5 & $181 \%$ \\
S3 & $40 \mathrm{mV}$ & 9.43 & 22.05 & $134 \%$ \\
S4 & $45 \mathrm{mV}$ & 6.9 & 17.09 & $148 \%$ \\
S5 & $50 \mathrm{mV}$ & 7.05 & 21.3 & $202 \%$ \\
S6 & $55 \mathrm{mV}$ & 4.79 & 18.4 & $284 \%$ \\
\hline \hline
\end{tabular}

dimension decreases at higher fill factors due to decreasing air void size compared to micropillar size. It can be concluded from the experimental results that increasing the micropillar

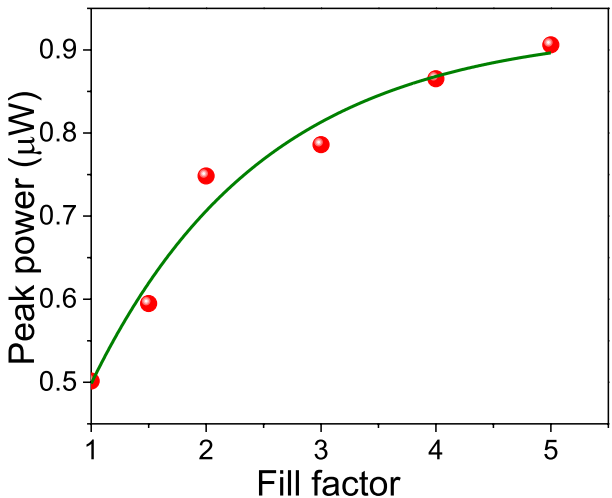

Fig. 13. Peak power generated for different micropillar arrays.

dimension results in increased contact area which results in increased power output but this effect starts diminishing at higher micropillar dimensions as the air voids becomes smaller leading to difficulty in triboelectric charge separation.

\section{CONClusion}

A novel design for contact electrification (triboelectric mechanism) based energy harvester is proposed and fabricated. A theoretical model has been developed to understand the deformation in SU-8 micropillars and PDMS layers. Simulations have been conducted to explain the working mechanism of TEH. Output voltage was observed to be increasing with increasing excitation acceleration. The peak power was obtained to be $0.91 \mu \mathrm{W}$ at $1 \mathrm{~g}$ and $24.5 \mathrm{~Hz}$ with a load resistance of $400 \mathrm{k} \Omega$. One of the main advantages of the presented design is broadband behavior observed in the resonant TEH due to non-linearity introduced by contact-separation 
mechanism. The bandwidth is observed to be continuously increasing as the acceleration level is increased.

The power output is found to be dependent on the size of micropillars and air voids between micropillars. As the micropillar dimension increases the power output increases due to increase in contact area between two surfaces. But this effect starts decreasing at higher fill factors i.e. higher micropillar dimensions due to decrease in the size of air voids which results in increased difficulty in triboelectric charge separation.

\section{ACKNOWLEDGMENTS}

Authors would like to thank Mathura Priyadharshini Thambidurai for her help in the experiments.

\section{REFERENCES}

[1] J. Paradiso and T. Starner, "Energy scavenging for mobile and wireless electronics," IEEE Pervas. Comput., vol. 4, no. 1, pp. 18-27, Mar. 2005.

[2] C. Enz, A. El-Hoiydi, and J. Decotignie, "WiseNET: An ultralow-power wireless sensor network solution," Computer, vol. 37, no. 8, pp. 62-70, Aug. 2004.

[3] V. Raghunathan, C. Schurgers, S. Park, and M. B. Srivastava, "Energyaware wireless microsensor networks," IEEE Signal Process. Mag., vol. 19, no. 2, pp. 40-50, Mar. 2002.

[4] S. J. Roundy, "Energy scavenging for wireless sensor nodes with a focus on vibration to electricity conversion," Dept. Mech. Eng., Univ. California, Berkeley, CA, USA, Tech. Rep., 2003.

[5] S. Roundy and P. K. Wright, "A piezoelectric vibration based generator for wireless electronics," Smart Mater. Struct., vol. 13, no. 5, pp. 1131-1142, 2004.

[6] S. Roundy, P. K. Wright, and J. Rabaey, "A study of low level vibrations as a power source for wireless sensor nodes," Comput. Commun., vol. 26, no. 11, pp. 1131-1144, Jul. 2003.

[7] M. Rasouli and L. S. J. Phee, "Energy sources and their development for application in medical devices," Expert Rev. Med. Devices, vol. 7, no. 5, pp. 693-709, 2010

[8] P. D. Mitcheson, E. M. Yeatman, G. K. Rao, A. S. Holmes, and T. C. Green, "Energy harvesting from human and machine motion for wireless electronic devices," Proc. IEEE, vol. 96, no. 9, pp. 1457-1486, Sep. 2008.

[9] A. Hajati and S.-G. Kim, "Ultra-wide bandwidth piezoelectric energy harvesting," Appl. Phys. Lett., vol. 99, no. 8, p. 083105, 2011.

[10] H. Liu, C. J. Tay, C. Quan, T. Kobayashi, and C. Lee, "Piezoelectric MEMS energy harvester for low-frequency vibrations with wideband operation range and steadily increased output power," J. Microelectromech. Syst., vol. 20, no. 5, pp. 1131-1142, 2011.

[11] R. Elfrink et al., "Vibration energy harvesting with aluminum nitridebased piezoelectric devices," J. Micromech. Microeng., vol. 19, no. 9, p. $94005,2009$.

[12] T. Galchev, E. Aktakka, and K. Najafi, "A piezoelectric parametric frequency increased generator for harvesting low-frequency vibrations," J. Microelectromech. Syst., vol. 21, no. 6, pp. 1311-1320, Dec. 2012.

[13] S. Priya, "Advances in energy harvesting using low profile piezoelectric transducers," J. Electroceram., vol. 19, no. 1, pp. 167-184, 2007.

[14] A. Abdelkefi, F. Najar, and A. Nayfeh, "An energy harvester using piezoelectric cantilever beams undergoing coupled bending-Torsion vibrations," Smart Mater. Struct., vol. 20, no. 11, p. 115007, 2011.

[15] M. Renaud, P. Fiorini, and C. Van Hoof, "Optimization of a piezoelectric unimorph for shock and impact energy harvesting," Smart Mater. Struct., vol. 16, no. 4, pp. 1125-1135, Aug. 2007.

[16] B. Yang et al., "Electromagnetic energy harvesting from vibrations of multiple frequencies," J. Micromech. Microeng., vol. 19, no. 3, p. 35001, 2009.

[17] S. P. Beeby et al., "A micro electromagnetic generator for vibration energy harvesting," J. Micromech. Microeng., vol. 17, no. 7, pp. 1257-1265, Jul. 2007.

[18] C. R. Saha, T. O’Donnell, N. Wang, and P. McCloskey, "Electromagnetic generator for harvesting energy from human motion," Sens. Actuators A, Phys., vol. 147, no. 1, pp. 248-253, Sep. 2008.
[19] S. Miki et al., "Electromagnetic energy harvester by using buried NdFeB," in Proc. IEEE 25th Int. Conf. MEMS, Feb. 2012 pp. 1221-1224.

[20] H. Liu, B. W. Soon, N. Wang, C. J. Tay, C. Quan, and C. Lee, "Feasibility study of a 3D vibration-driven electromagnetic MEMS energy harvester with multiple vibration modes," J. Micromech. Microeng., vol. 22, no. 12, p. 125020, Dec. 2012.

[21] P. Basset, D. Galayko, A. M. Paracha, F. Marty, A. Dudka, and T. Bourouina, "A batch-fabricated and electret-free silicon electrostatic vibration energy harvester," J. Micromech. Microeng., vol. 19, no. 11, p. 115025 , Nov. 2009.

[22] Y. Naruse, N. Matsubara, K. Mabuchi, M. Izumi, and S. Suzuki, "Electrostatic micro power generation from low-frequency vibration such as human motion," J. Micromech. Microeng., vol. 19, no. 9, p. 094002, Sep. 2009

[23] H. Lo and Y.-C. Tai, "Parylene-based electret power generators," J. Micromech. Microeng., vol. 18, no. 10, p. 104006, Oct. 2008.

[24] E. Halvorsen et al., "An electrostatic energy harvester with electret bias," in Proc. Int. Solid-State Sens., Actuators, Microsyst. Conf., 2009, pp. 1381-1384

[25] P. Bai et al., "Integrated multilayered triboelectric nanogenerator for harvesting biomechanical energy from human motions," ACS Nano, vol. 7, no. 4, pp. 3713-3719, Mar. 2013.

[26] T.-C. Hou, Y. Yang, H. Zhang, J. Chen, L.-J. Chen, and Z. L. Wang, "Triboelectric nanogenerator built inside shoe insole for harvesting walking energy," Nano Energy, vol. 2, no. 5, pp. 856-862, Sep. 2013.

[27] G. Zhu et al., "Toward large-scale energy harvesting by a nanoparticleenhanced triboelectric nanogenerator," Nano Lett., vol. 13, no. 2, pp. 847-853, Feb. 2013.

[28] F.-R. Fan, L. Lin, G. Zhu, W. Wu, R. Zhang, and Z. L. Wang, "Transparent triboelectric nanogenerators and self-powered pressure sensors based on micropatterned plastic films," Nano Lett., vol. 12, no. 6, pp. 3109-3114, Jun. 2012.

[29] X.-S. Zhang et al., "Frequency-multiplication high-output triboelectric nanogenerator for sustainably powering biomedical microsystems," Nano Lett., vol. 13, no. 3, pp. 1168-1172, 2013.

[30] J. Lowell and A. C. Rose-Innes, "Contact electrification," Adv. Phys., vol. 29, no. 6, pp. 947-1023, 1980.

[31] R. G. Horn and D. T. Smith, "Contact electrification and adhesion between dissimilar materials," Science, vol. 256, no. 5055, pp. 362-364, Apr. 1992.

[32] D. K. Davies, "Charge generation on dielectric surfaces," J. Phys. D, Appl. Phys., vol. 2, no. 11, pp. 1533-1537, 1969.

[33] J. Henniker, "Triboelectricity in polymers," Nature, vol. 196, p. 474, Nov. 1962.

[34] S. Matsusaka, H. Maruyama, T. Matsuyama, and M. Ghadiri, "Triboelectric charging of powders: A review," Chem. Eng. Sci., vol. 65, no. 22 , pp. 5781-5807, Nov. 2010

[35] I. Sari, T. Balkan, and H. Kulah, "An electromagnetic micro power generator for wideband environmental vibrations," Sens. Actuators A, Phys., vol. 146, pp. 405-413, Aug. 2008.

[36] L. G. W. Tvedt, D. S. Nguyen, and E. Halvorsen, "Nonlinear behavior of an electrostatic energy harvester under wide- and narrowband excitation," J. Microelectromech. Syst., vol. 19, no. 2, pp. 305-316, 2010.

[37] V. R. Challa, M. G. Prasad, Y. Shi, and F. T. Fisher, "A vibration energy harvesting device with bidirectional resonance frequency tunability," Smart Mater. Struct., vol. 17, no. 1, p. 15035, 2008.

[38] M. S. M. Soliman, E. M. Abdel-Rahman, E. F. El-Saadany, and R. R. Mansour, "A wideband vibration-based energy harvester," J. Micromech. Microeng., vol. 18, no. 11, p. 115021, Nov. 2008.

[39] H. Liu, C. Lee, T. Kobayashi, C. J. Tay, and C. Quan, "Investigation of a MEMS piezoelectric energy harvester system with a frequency-widenedbandwidth mechanism introduced by mechanical stoppers," Smart Mater. Struct., vol. 21, no. 3, p. 035005, Mar. 2012.

[40] M. Soliman, E. M. Abdel-Rahman, E. F. El-Saadany, and R. R. Mansour, "A design procedure for wideband micropower generators," J. Microelectromech. Syst., vol. 18, no. 6, pp. 1288-1299, Dec. 2009.

[41] H. Liu, C. Lee, T. Kobayashi, C. J. Tay, and C. Quan, "Piezoelectric MEMS-based wideband energy harvesting systems using a frequencyup-conversion cantilever stopper," Sens. Actuators A, Phys., vol. 186, pp. 242-248, Oct. 2012.

[42] L. Dhakar, H. Liu, F. E. H. Tay, and C. Lee, "A new energy harvester design for high power output at low frequencies," Sens. Actuators A, Phys., vol. 199, pp. 344-352, Sep. 2013.

[43] W. J. Stronge, Impact Mechanics. Cambridge, U.K.: Cambridge Univ. Press, 2000 
[44] S. Matsusaka, M. Ghadiri, and H. Masuda, "Electrification of an elastic sphere by repeated impacts on a metal plate," J. Phys. D, Appl. Phys., vol. 33, no. 18 , pp. 2311-2319, Sep. 2000

[45] S. H. Crandall, T. J. Lardner, and N. C. Dahl, An Introduction to the Mechanics of Solids: With SI Units. New York, NY, USA: McGraw-Hill, 1999.

[46] S. C. B. Mannsfeld et al., "Highly sensitive flexible pressure sensors with microstructured rubber dielectric layers," Nature Mater., vol. 9, no. 10 , pp. 859-864, 2010.

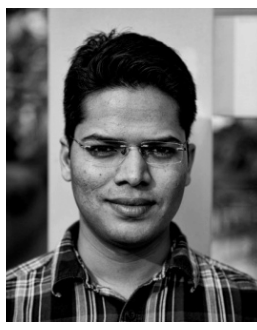

Lokesh Dhakar received his B.E. (Hons.) degree in Mechanical Engineering from Birla Institute of Technology and Science, Pilani, in 2010. He worked in the industry as a design engineer from 2010 to 2011. He is currently working towards his Ph.D. degree from NUS Graduate School of Integrative Sciences and Engineering, National University of Singapore, Singapore. He is concurrently also pursuing his M.B.A. from NUS Business School. He is keenly interested in entrepreneurship and technology commercialization. He is currently working on energy harvesting devices aimed at powering wireless wearable sensors for healthcare.

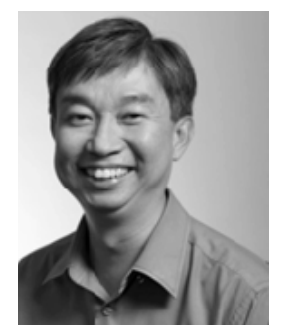

Francis Eng Hock Tay is an Associate Professor with the Department of Mechanical Engineering, Faculty of Engineering, National University of Singapore. He received the $\mathrm{Ph} . \mathrm{D}$. degree from the Massachusetts Institute of Technology (MIT), Cambridge, in 1995. Dr. Tay was the founding director of the Microsystems Technology Initiative (MSTI), and had established the Microsystems Technology Specialization. He has also served as the Technical Advisor in the Micro and Nano Systems Laboratory, Institute of Materials Research Engineering (IMRE). His research areas include MEMS, biotechnology, nanotechnology, wearable devices, fall onset detection, vital signs monitoring, body sensor network, rehabilitation, and scoliosis. He is also the Principal Investigator for several projects under the Agency for Science, Technology, and Research (A*STAR), and one project under the Qatar National Research Fund (QNRF).

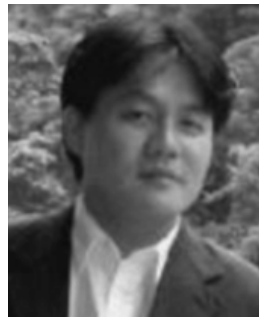

Chengkuo Lee (S'93-M'96) received the M.S. degree in materials science and engineering from National Tsing Hua University, Hsinchu, Taiwan, in 1991, the M.S. degree in industrial and system engineering from Rutgers University, New Brunswick, NJ, in 1993, and the Ph.D. degree in precision engineering from The University of Tokyo, Tokyo, Japan, in 1996. He worked as a Foreign Researcher in the Nanometerscale Manufacturing Science Laboratory of the Research Center for Advanced Science and Technology, The University of Tokyo, from 1993 to 1996. He also worked in the Mechanical Engineering Laboratory, AIST, MITI of Japan as a JST Research Fellow in 1996. Thereafter, he became a Senior Research Staff Member of the Microsystems Laboratory, Industrial Technology Research Institute, Hsinchu, Taiwan. In September 1997, he joined Metrodyne Microsystem Corporation, Hsinchu, Taiwan, and established the MEMS device division and the first micromachining fab for commercial purposes in Taiwan. He was the Manager of the MEMS device division between 1997 and 2000. He was an Adjunct Assistant Professor in the Electrophysics Department of National Chiao Tung University, Hsinchu, Taiwan, in 1998, and an Adjunct Assistant Professor in the Institute of Precision Engineering of National Chung Hsing University, Taichung, Taiwan, from 2001 to 2005. In August 2001, he cofounded Asia Pacific Microsystems, Inc. (APM), where he first became Vice President of R\&D, before becoming Vice President of the optical communication business unit and Special Assistant to the Chief Executive Officer in charge of international business and technical marketing for the MEMS foundry service. From 2006 to 2009, he was a Senior Member of the Technical Staff at the Institute of Microelectronics, A-STAR, Singapore. Currently he is an associate Professor in the Department of Electrical and Computer Engineering, National University of Singapore, Singapore. He is the coauthor of Advanced MEMS Packaging (McGrawHill, 2010). He has contributed to more than 200 international conference papers and extended abstracts, and 150 peer-reviewed international journal articles in the fields of sensors, actuators, energy harvesting, MEMS, NEMS, nanophotonics, and nanotechnology. He is also the holder of nine U.S. patents. 